\title{
Mapping the Local Spatial Charge in Defective Diamond by Means of N-V Sensors-A Self-Diagnostic Concept
}

\author{
J. Forneris,,${ }^{1, *}$ S. Ditalia Tchernij, ${ }^{2,1}$ P. Traina, ${ }^{3}$ E. Moreva, ${ }^{3}$ N. Skukan, ${ }^{4}$ M. Jakšić, ${ }^{4}$ V. Grilj, ${ }^{4}$ \\ F. Bosia, ${ }^{2,1}$ E. Enrico, ${ }^{3}$ G. Amato, ${ }^{3}$ I.P. Degiovanni, ${ }^{3}$ B. Naydenov,${ }^{5}$ F. Jelezko,${ }^{5}$ M. Genovese,${ }^{3,1}$ and \\ P. Olivero ${ }^{2,1}$ \\ ${ }^{1}$ Istituto Nazionale di Fisica Nucleare (INFN), Sez. Torino, via P. Giuria 1, 10125 Torino, Italy \\ ${ }^{2}$ Physics Department and "NIS" Inter-Departmental Centre, Università di Torino, via P. Giuria 1, 10125 Torino, \\ Italy \\ ${ }^{3}$ Istituto Nazionale di Ricerca Metrologica (INRiM), Strada delle Cacce 91, 10135 Torino, Italy \\ ${ }^{4}$ Ruđer Bošković Institute, Bijenicka 54, P.O. Box 180, 10002 Zagreb, Croatia \\ ${ }^{5}$ Institute for Quantum Optics and Center for Integrated Quantum Science and Technology (IQST), \\ Albert-Einstein-Allee 11, Universität Ulm, D-89069 Ulm, Germany
}

(Received 26 June 2017; revised manuscript received 4 April 2018; published 25 July 2018)

\begin{abstract}
Electrically active defects have a significant impact on the performance of electronic devices based on wide-band-gap materials. This issue is ubiquitous in diamond science and technology, since the presence of charge traps in the active regions of different classes of diamond-based devices (detectors, power diodes, transistors) can significantly affect their performance, due to the formation of space charge, memory effects, and the degradation of the electronic response associated with radiation-induced damage. Among the most common defects in diamond, the nitrogen-vacancy $(\mathrm{N}-V)$ center possesses unique spin properties that enable high-sensitivity field sensing at the nanoscale. Here, we demonstrate that $\mathrm{N}-V$ ensembles can be successfully exploited to perform direct local mapping of the internal electric-field distribution of a graphite-diamond-graphite junction exhibiting electrical properties dominated by trap- and space-chargerelated conduction mechanisms. By means of optically detected magnetic resonance measurements, we performed both point-by-point readout and spatial mapping of the electric field in the active region at different bias voltages. In this novel "self-diagnostic" approach, defect complexes represent not only the source of detrimental space-charge effects but also a unique tool for their direct investigation, by providing an insight on the conduction mechanisms that could not be inferred in previous studies on the basis of conventional electrical and optical characterization techniques.
\end{abstract}

DOI: 10.1103/PhysRevApplied.10.014024

\section{INTRODUCTION}

Diamond is an appealing material for the development of innovative devices, such as high-power and fast electronics [1-4], radiation dosimeters [5,6] and detectors [7,8], biosensors [9-11], and, more recently, integrated platforms for quantum technologies [10-15]. For these applications, a major issue to be addressed in the optimization of device performance is represented by the effect of lattice defects with deep levels in the diamond energy gap acting as charge carrier traps. It has been extensively reported that the introduction of carrier traps caused by the interaction with energetic radiation induces electric-field inhomogeneities and polarization or memory effects in the material [16-18]. In previous works, these effects have been widely investigated by the analysis of current-voltage characteristics exhibiting complex

*forneris@to.infn.it
non-Ohmic behavior, elucidating several conduction models ranging from space-charge-limited current (SCLC) to Poole-Frenkel (PF) mechanisms [19-22]. Complementarily, ion, electron, x-ray, and visible beam-induced charge microscopies allow the mapping of the chargetransport parameters of diamond at the microscale [18, 23-26]. Although insightful, these techniques cannot provide direct and unambiguous experimental evidence of the local electric-field distribution in the defective material but, rather, need to model it by means of simplified finite-element methods. Among the large variety of lattice defects in diamond, the nitrogen-vacancy complex (N- $V$ center) has emerged as a system characterized by unique spin properties, enabling magnetic-, thermal-, and electric-field sensing with high sensitivity and spatial resolution [27-32]. The very same $\mathrm{N}-V$ centers that are created (among other types of defect complexes) by radiation damage [33] can be therefore exploited to locally investigate the internal electric-field distribution 
(a)

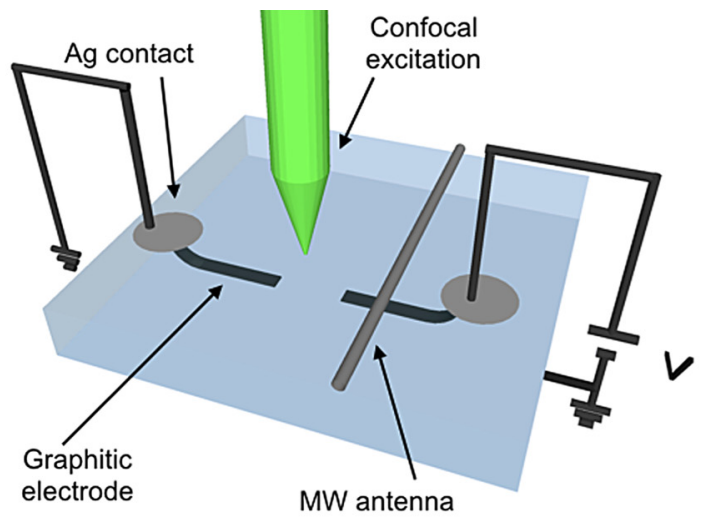

(c)

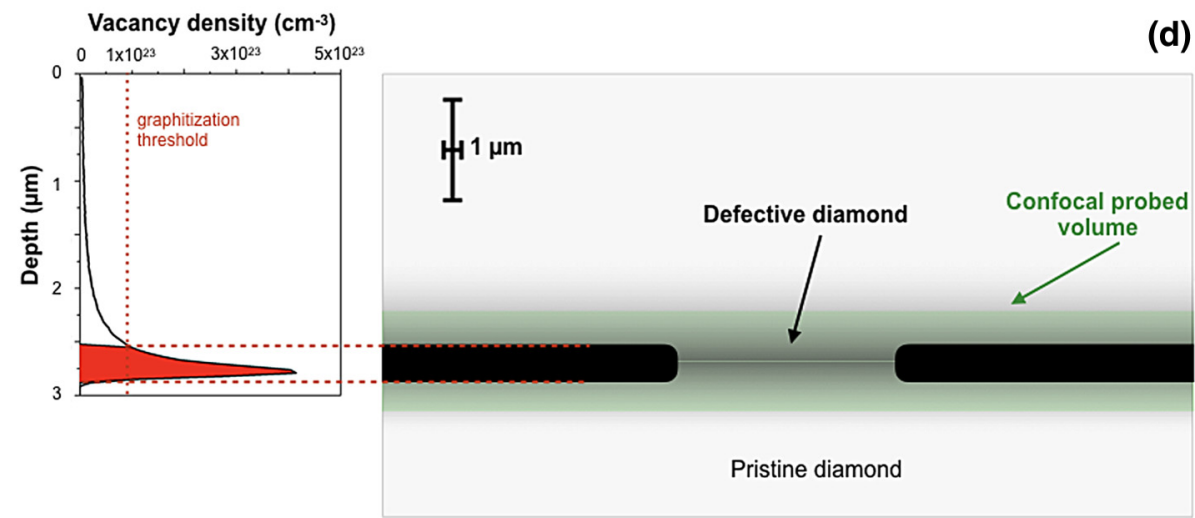

(b)

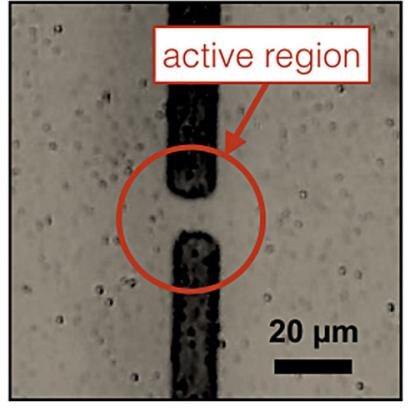

(d) of the irradiated devices [32,34]. In this work, we show that the optically detected magnetic resonance (ODMR) readout of the electronic spin from ensembles of $\mathrm{N}-V$ centers can be usefully exploited to map the internal electric-field distribution in defective diamond, in order to diagnose the effects of electrically active traps. For this purpose, we employed a graphite-diamond-graphite junction fabricated by means of $\mathrm{MeV}$ ion-beam lithography [22]. Strong local electric fields were applied in the micrometer-sized diamond region between the graphitic electrodes, containing a $\sim 10^{15} \mathrm{~cm}^{-3}$ density of radiation-induced defects, including electrically active carrier traps. In this self-diagnostic approach, it was thus possible to investigate the space-charge buildup and the local electric-field distribution under different biasing conditions.

\section{DEVICE FABRICATION AND EXPERIMENTAL SETUP}

We investigated a graphite-diamond-graphite junction embedded in a chemical-vapor-deposited optical-grade type-IIa single-crystal substrate supplied by ElementSix Ltd, cut along the $\langle 100\rangle$ orientation [Fig. 1(a)]. The structure consists of two $\sim 15-\mu \mathrm{m}$-wide $100-\mu \mathrm{m}$-long graphitic microelectrodes elongated in the $\langle 100\rangle$ crystal direction, whose endpoints are spaced by a $\sim 9-\mu \mathrm{m}$ active region [an optical micrograph is shown in Fig. 1(b)]. The electrodes were fabricated in a planar geometry by raster-scanning
FIG. 1. (a) Schematic representation (not to scale) of the graphite-diamond-graphite junction. (b) Optical micrograph of the junction. The active region between the graphitic electrodes is highlighted. (c) Monte Carlo simulation of the vacancy-density depth profile of diamond irradiated with $6-\mathrm{MeV}$ $\mathrm{C}$ ions at a fluence of $4 \times 10^{16}$ $\mathrm{cm}^{-2}$ [35]. The region highlighted in red corresponds to the layer converted to a graphitic phase upon thermal annealing of the irradiated substrate. (d) Schematic representation (to scale) of the crosssection of the device, highlighting the depth and thickness of the graphitic electrodes and the presence of a defective region in the active region. The region highlighted in green indicates the $\sim 1-\mu$ m-thick focal plane probed by the confocal microscope. a $\sim 5-\mu$ m-sized $6-\mathrm{MeV}-\mathrm{C}^{3+}$ beam, as explained in detail in previous works $[22,35]$. The implantation fluence was chosen to ensure the amorphization of a diamond layer at the end of the ion range [ 2.7 $\mu \mathrm{m}$; see Fig. 1(c)] [36], where the irradiation-induced vacancy density exceeded a graphitization threshold [37,38] for $\mathrm{MeV}$ ions, resulting in the conversion of a $\sim 0.5-\mu \mathrm{m}$-thick layer to a graphitic phase by a subsequent thermal annealing. Further experimental details on the fabrication process are given in Appendix C.

The ion microbeam fabrication also resulted in the implantation of a halo of stray ions surrounding the graphitized layers [Fig. 1(d)] and located mainly on a plane at the same depth as that of the graphitic electrodes. The size of such a defect distribution is comparable with the active region and associated with a vacancy density of $\sim 1 \times 10^{22}$ $\mathrm{cm}^{-3}$, as estimated in previous works $[22,35]$. Such a value is below the graphitization threshold for subsuperficial ion implantations but high enough to promote the formation of a high concentration of $\mathrm{N}-V$ centers upon thermal annealing [33], as well as to introduce other types of defects and carrier traps, such as the $B$-band and interstitial-related defects [22]. The $\mathrm{N}-V$ concentration is estimated to be proportional to the density of vacancies introduced in the diamond lattice [see Fig. 1(c)] and to be of the order of $10^{3}-10^{4}$ centers $/ \mu \mathrm{m}^{3}$ at the end of the ion range in diamond [22]. This value has to be compared with the typical concentration of $\sim 10^{2}$ centers $/ \mu \mathrm{m}^{3}$ in a pristine opticalgrade substrate [39]. The lattice structure was not fully 
recovered by the thermal treatment and the overall trap concentration in the junction was quantified as $\sim 2 \times 10^{15}$ $\mathrm{cm}^{-3}$ by previous electrical characterization [35].

Photoluminescence (PL) and ODMR measurements were performed by mapping the graphite-diamondgraphite junction across a plane parallel to the sample surface. The focal plane was set to coincide with the end of the $6-\mathrm{MeV} \mathrm{C}^{3+}$ range, i.e., where the concentration of radiation-induced defects and $\mathrm{N}-V$ centers is maximal. The measured signal was therefore averaged along a focal depth of $\sim 1 \mu \mathrm{m}$, i.e., comparable with the thickness of the graphitic electrodes [see Fig. 1(d)].

\section{RESULTS AND DISCUSSION}

\section{A. Electrical characterization}

The room-temperature current-voltage characteristics of the junction are presented in Fig. 2 in the same voltage ranges as explored in the ODMR measurements [22,35]. The curve exhibits an Ohmic trend up to a voltage threshold $\left(V_{T} \sim+250 \mathrm{~V}\right)$, at which the space-charge density starts affecting the conduction mechanism and a deviation from the linear trend is observed. In accordance with the SCLC model [22,34], this deviation is associated with the progressive filling of traps by the electrons injected in the active region [38] and leads to the transition to a high-current regime $(>10 \mu \mathrm{A})$ at voltages larger than a critical value $V_{C}(\sim+330 \mathrm{~V})$. At higher bias voltages, the superlinear dependence of the current on the applied bias is compatible with the PF conduction model [40].

According to the SCLC model, the current is limited at biases in the $V_{T}<V<V_{C}$ range by a counter-field built by the progressive filling of traps by carriers injected in the active region of the junction [41]. Such an interpretation is based on a unipolar conduction mechanism [42] and

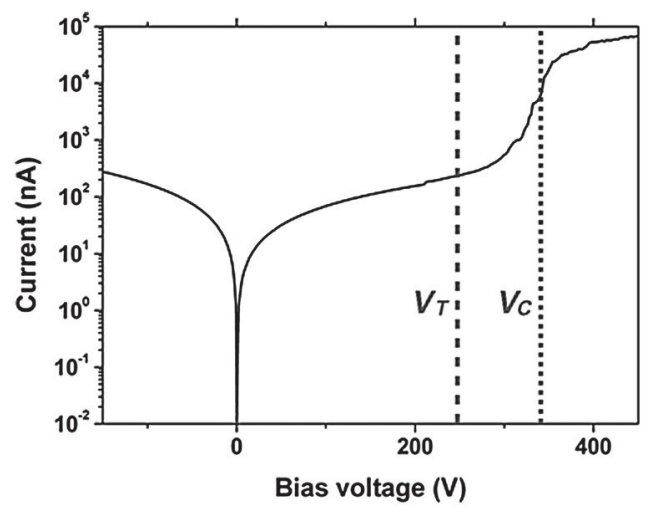

FIG. 2. Current-voltage characteristic of the junction; the threshold and critical bias voltages ( $V_{T}$ and $V_{C}$, respectively) are marked by dashed vertical lines. is consistent with several experimental features observed over repeated measurement cycles, i.e., different slopes in the current-voltage curves under polarity reversal (Fig. 2), a progressive conversion of the $\mathrm{N}-V$ centers to a negative charge state at increasing currents [22], a decrease in the critical voltage at increasing temperatures [35], a marginal variability of a few volts in the value of $V_{C}$ from different experimental runs [35], and a pronounced current-voltage hysteresis at decreasing bias voltages [15].

\section{B. ODMR spectra at variable applied bias voltage}

The PL mapping at zero bias voltage of the regions located above the electrodes [Fig. 3(a)] exhibits an intense emission from the regions directly above the electrodes, which is determined by $\mathrm{N}-V$ centers and other optically active radiation-induced defects located between the sample surface and the graphitic layer. Conversely, the PL emission from the active region is largely dominated by $\mathrm{N}-V$ ensembles [22]. The peculiar spin properties of the negatively charged $\mathrm{N}-V$ center were exploited to obtain a direct measurement of the local electric field in the active region of the device by means of ODMR. The technique is extensively described in Refs. [43-49], and the basic principles applied in this work to electric-field sensing are summarized in Appendix A.

Before the ODMR measurements, the junction underwent multiple voltage sweeps in the 0 to $+200 \mathrm{~V}$ range, resulting in the buildup of a space charge in the active region. In Fig. 3(e), the label [C] identifies the initial "charged" state of the junction at zero bias voltage. Subsequently, ODMR spectra were acquired at increasing bias voltages from 0 to $+350 \mathrm{~V}$ at the fixed position labeled by the green spot in Fig. 3(a): this first voltage sweep is indicated in Fig. 3(e) by the arrow labeled as (1). A second bias-voltage sweep from 0 to $-105 \mathrm{~V}$ [labeled as (3)] was subsequently acquired from the same spot after the bias voltage had reverted to zero [label (2)]. Each ODMR spectrum displays two dips at the characteristic resonance frequencies for $\mathrm{N}-V^{-}$centers, with a splitting value that is systematically dependent on the strength of the local electric field, as is clearly visible in Fig. 3(b), which reports in color scale (encoding the normalized PL intensity) the series of acquired ODMR spectra as a function of the bias voltage. The observation of two resonances in the recorded ODMR spectra is consistent with the geometry of the junction, since the electric field within the active region is mainly parallel to the axis joining the graphitic electrodes, i.e., the $\langle 100\rangle$ crystal direction [Fig. (3a)]. It is worth noting that the ODMR spectra were acquired from a $\sim 1-\mu$ m-thick focal plane centered at the electrode depth. For this reason, the ODMR measurement results from an average of the electric field sensed by an ensemble of $\mathrm{N}-V$ centers over the above-mentioned depth. 
(a)

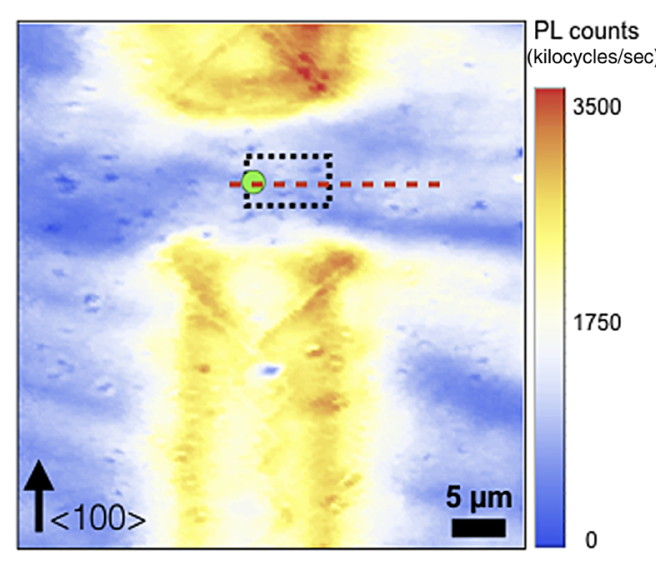

(c)

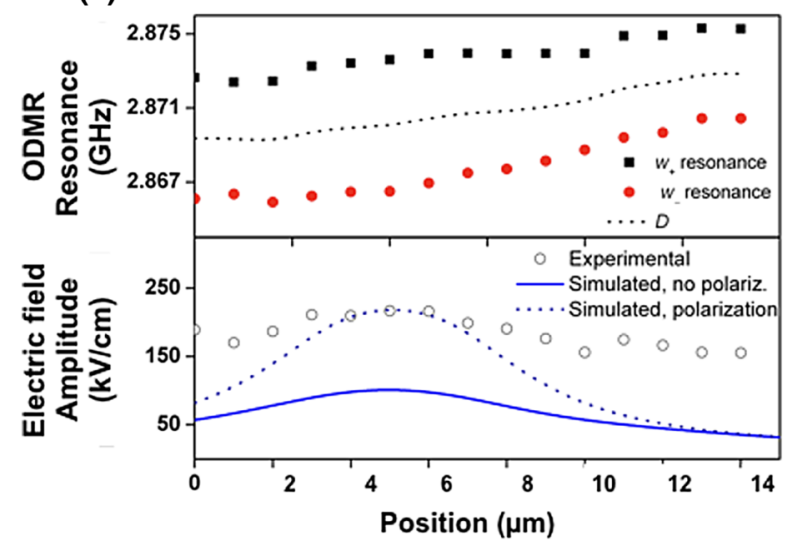

(b)

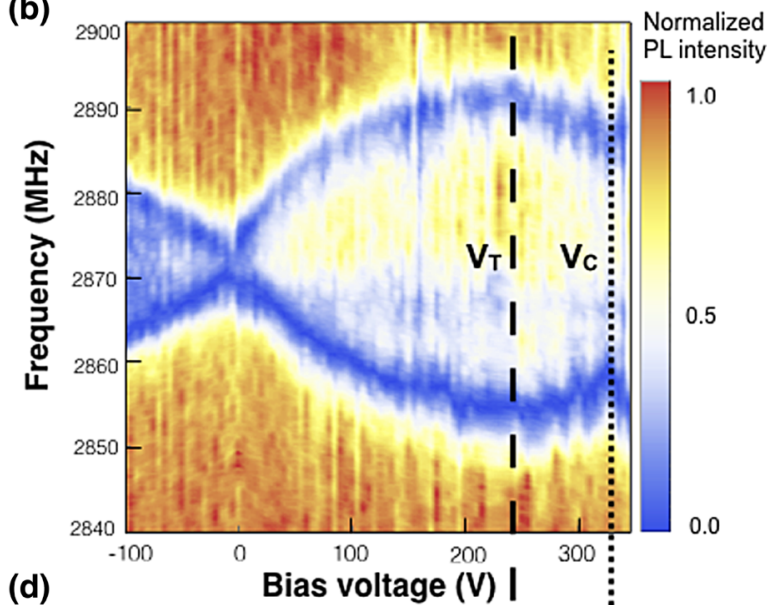

(d)

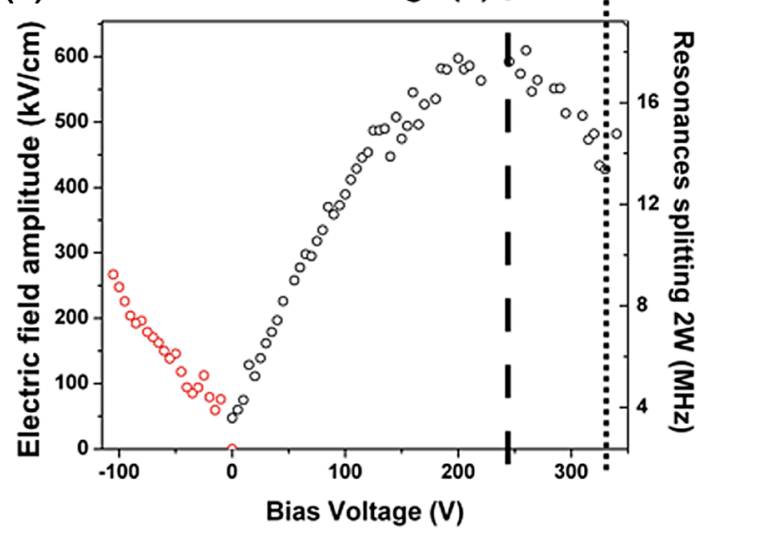

(1)

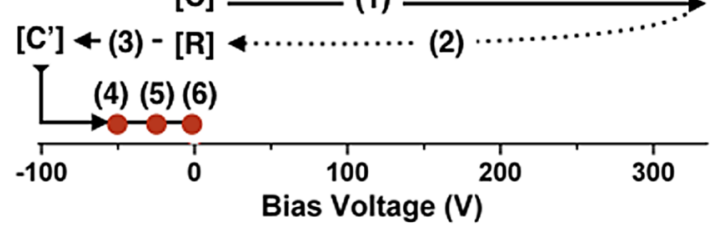

FIG. 3. (a) PL map of the unbiased junction; the rectangular region highlighted by the dashed black line indicates the region where the electric-field maps displayed in Fig. 6 were acquired; the green spot indicates the point where the ODMR spectra under applied voltage displayed in (b) and (d) were acquired; the dashed red line indicates the scan along which the electric-field profile displayed in (c) was measured. (b) Intensity-color-encoded and normalized ODMR spectra acquired during a voltage sweep from the position highlighted by the green spot in (a). The black dashed line indicates the bias voltages $V_{T}$ corresponding to which the current-voltage characteristic deviates from the linear trend (see Fig. 2). The black dotted line indicates the bias voltage $V_{C}$ corresponding to the transition to the high-current regime (see Fig. 2). (c) ODMR resonances (top, black, and red data points) acquired along the red dashed line highlighted in (a) at a bias voltage of $-50 \mathrm{~V}$. The dashed black line indicates the evaluated $D$ frequency. The electric-field profile extracted from the experimental data (bottom, black circles) is reported together with its numerical simulations based on a two-dimensional FEM model in the presence of a local space charge, as discussed in Sec. III C. The blue line indicates the simulated profile obtained assuming a space-charge extension $u(V=-50 \mathrm{~V})=2.5 \mu \mathrm{m}$, in agreement with the data in Fig. 5(a). The blue dotted line represents the simulated profile obtained including an additional space-charge effect due to diamond polarization, i.e., assuming $u(V=-50 \mathrm{~V})=3 \mu \mathrm{m}$. (d) Electric-field amplitude vs the applied bias voltage, as evaluated from the ODMR splitting measured at the point highlighted in green in (a). (e) Layout of the voltage bias cycles performed on the junction and discussed in Secs. III B and III C. The arrows indicate the temporal sequence of the operations.

In order to interpret the ODMR spectra according to the Hamiltonian discussed in Appendix A, as well as to provide an assessment of the electric field outside of the central region, an ODMR scan was subsequently acquired at $-50 \mathrm{~V}$ applied bias [label (4) in Fig. 3(e)] along a $15-\mu \mathrm{m}$-long horizontal line [highlighted by the red dashed 
line in Fig. 3(a)] bisecting the active area of the junction. The relevant fitted values of the ODMR resonances are reported in Fig. 3(c).

The ODMR resonances were analyzed to estimate the amplitude of the electric field according to the theoretical framework reported in Eqs. (A3) and (A4) of Appendix A. It is worth remarking that, as discussed above, the ODMR resonance frequencies depend on both the amplitude of the electric field and other additional effects, such as (i) background magnetic fields, due to the fact that the experimental setup was not shielded (ii) local temperature variations related to the injected current of the device and (iii) local stress fields due to the mechanical distortion of the diamond substrate related to the previous ion implantation. These contributions to the measured ODMR signals were suitably considered and deconvoluted from the effect of interest in the present work, i.e., the mapping of the electric-field distribution, as detailed in Appendix B. The following section reports on the interpretation of the obtained results upon such deconvolution.

\section{Data discussion and numerical simulations}

\section{Voltage-dependent electric field at the center of the active region}

The transverse component of the electric field at the probed spot was evaluated from the ODMR spectra according to the procedure discussed in Appendix B, upon the deconvolution of concurrent effects of background magnetic fields and local temperature or stress variations. Considering that the electric field is oriented along the $\langle 100\rangle$ crystallographic direction, the four possible orientations of the $\mathrm{N}-V$ defects form a $\alpha=54.75^{\circ}$ angle with the electric field, and thus a fourfold degeneracy in the respective spin resonances is expected. This observation is in line with the presence of only two resonances in the ODMR spectra in Fig. 3(b), indicating the degeneracy in the projection of the electric-field vector on all the $\mathrm{N}-V$ center orientations. The amplitude of the electric-field vector has therefore been calculated as $|E|=E_{\perp} / \sin (\alpha)$.

The evaluated values of the electric-field amplitude are reported in Fig. 3(c) for the horizontal profile along the symmetry axis of the junction, and in Fig. 3(d) for the voltage-dependent measurements acquired from the probed spot at the center of the active region. The same evaluation procedure has also been adopted to define the electric-field maps discussed in the last section of this work. Figure 3(d) reports the electric-field amplitude at the point highlighted in green in Fig. 3(a) as a function of the applied bias voltage. The scale on the right-hand vertical axis indicates the evaluated resonance splitting $2 W_{E}=$ $2\left\{-k_{\perp} P+\left[W^{2}-\left(\mu g B_{\|}\right)^{2}\right]^{1 / 2}\right\}$ associated with the nonaxial electric-field contribution, upon the deconvolution of magnetic and stress fields discussed in Appendix A. For applied bias voltages below $V_{T}$, the electric field linearly increases with the applied bias voltage at both polarities, although starting from a nonzero value at the zero bias voltage in the [C] configuration of the device [see Fig. 3(e)]. However, its trend is not symmetric under polarity reversal, in accordance with the nonsymmetrical behavior of the current-voltage characteristics shown in Fig. 1(c).

After a progressive deviation from the linear behavior observed in the $+125-245-\mathrm{V}$ range, the estimated local electric field reaches a maximum value at positive bias voltages ranging between $V_{d / 2} \sim 200 \mathrm{~V}$ and $V_{T} \sim 245 \mathrm{~V}$. At higher bias voltages, the electric field steadily decreases up to $V \sim V_{C}$. While the few data points acquired at bias voltages higher than $V_{C}$ suggest a new increase [Figs. 3(b)-3(d)], the voltage dependence of the electric field could not be adequately assessed in this conduction regime, due to the concurrent electroluminescent emission occurring from neutrally charged $\mathrm{N}-V^{0}$ centers [22], which significantly decreased the ODMR contrast.

The overall nonmonotonic trend provides a direct insight into the conduction mechanism occurring in the active region [40]. In order to gain a deeper insight into the operation of the junction, the experimental data acquired at the central position of the active region [i.e., from the point highlighted in green in Fig. 3(a)] were compared in Fig. 4(a) (black circles) to the results of a one-dimensional finite-element-method (FEM) simulation (black line) based on the solution of Laplace's equation. This numerical result was obtained by assuming the absence of internal space charge and was performed assuming a relative dielectric constant $\varepsilon_{r}=7.6$ for the defective substrate, as estimated in a previous electrical characterization of the same device [35].

The electric-field estimation at positive bias voltages (i.e., the first voltage sweep) differs significantly from the FEM numerical predictions, suggesting that the preliminary voltage sweeps carried on the sample before this measurement produced a space-charge buildup near the cathode, resulting in a significant increase of the internal electric field under positive polarity. The contribution of the space charge to the electric field [Fig. 4(a), green circles], estimated as the difference between the electricfield amplitude evaluated from ODMR measurements and the corresponding FEM-simulated value, has the same nonmonotonic trend at increasing bias voltage, with a maximum centered at $V \sim V_{d / 2}$. This trend was interpreted according to the simple model of the space-charge buildup schematically represented in Fig. 4(b). While the absence of local charge buildup is schematically represented in Fig. 4(b,I), according to this model the extension of the space charge $u(V)$ increases with the bias voltage from the cathode to the anode due to the progressive trapping of electrons injected in the defective diamond. At low bias voltages (i.e., at $V<V_{d / 2}$ ), $u(V)$ is smaller than $d / 2$, which corresponds to the distance between the probed spot 
(a)

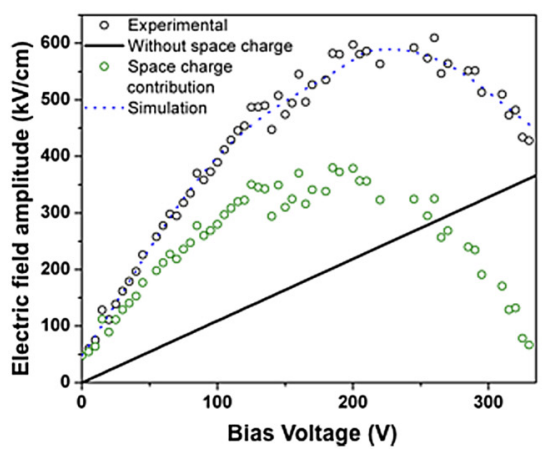

(b)

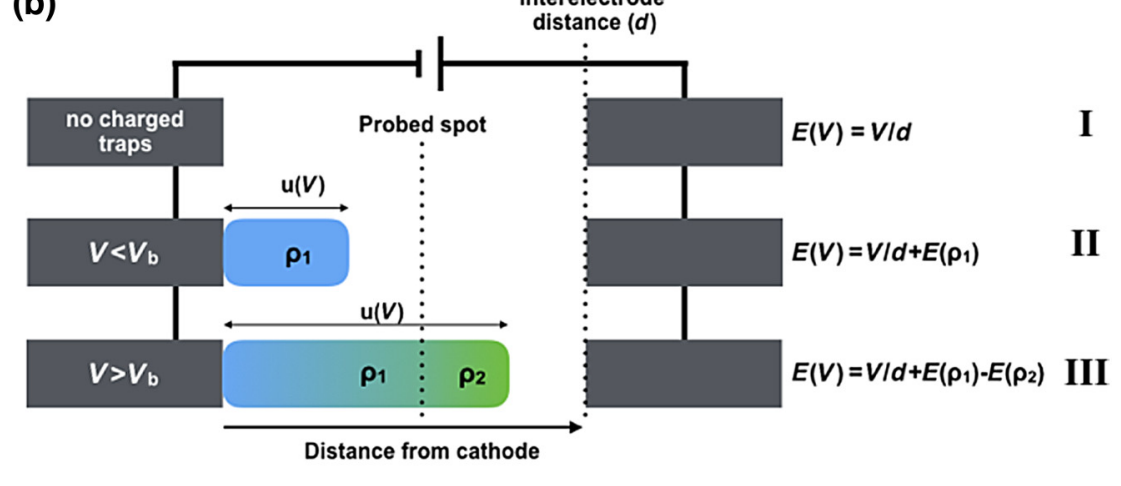

FIG. 4. (a) Voltage-dependent electric-field amplitude as estimated from: (i) the ODMR spectra reported in Fig. 3(b) [black circles, same data as in Fig. 3(d)] and (ii) a one-dimensional FEM numerical simulation in the absence of an internal space charge (black line). The difference between the two trends (green circles) represents the electric field associated with the presence of an internal space charge. The residual nonzero value of the electric field at zero bias voltage [configuration labeled as [C] in Fig. 3(e)] is a consequence of previous biasing cycles performed on the junction. The dashed blue line represents the trend of the electric field as evaluated by finite-element-method simulations. (b) Simplified model describing the contribution of a voltage-dependent internal space charge on the electric field measured at the probed spot [green spot in Fig. 3(a)]. The presence of a space-charge distribution $\rho_{1}$ between the cathode and the probed spot boosts the internal electric field (II) with respect to the uncharged junction (I). An additional space charge $\rho_{2}$ between the probed spot and the anode (III) results in a partial screening of the electric field generated by the bias voltage and the space charge $\rho_{1}$ between the cathode and the probed spot.

and the cathode, $d$ being the interelectrode distance [Fig. $4(\mathrm{~b}, \mathrm{II})]$. As a consequence, the associated negative spacecharge density $\rho_{1}$ produces an incremental electric field $E\left(\rho_{1}\right)$ adding up to that generated by the bias voltage. Conversely, when the extension of the space charge $u(V)$ is larger than $d / 2$ [Fig. 4(b,III)], the internal electric field is both incremented by the contribution $E\left(\rho_{1}\right)$ generated by the fully charged region at $x<d / 2$ and screened by the term $E\left(\rho_{2}\right)$ related to the negative charge $\rho_{2}$ progressively extending between the probed spot $(x=d / 2)$ and the anode $(x>d / 2)$.

\section{Space-charge modeling}

The suitability of the above-described model to provide an adequate description of the ODMR data acquired at positive bias voltages was assessed by including a space-charge distribution in the FEM simulations. The source term $\rho$ of the relevant Poisson's equation was defined assuming, for simplicity, that the space-charge buildup results in the complete electron filling of a uniform distribution of traps $N_{T}$, with a gradually increasing extension $u(V)$ from the cathode [Fig. 4(b)]. The $u(V)$ extension was therefore modeled with a step function $\Theta(x)$, and (a)

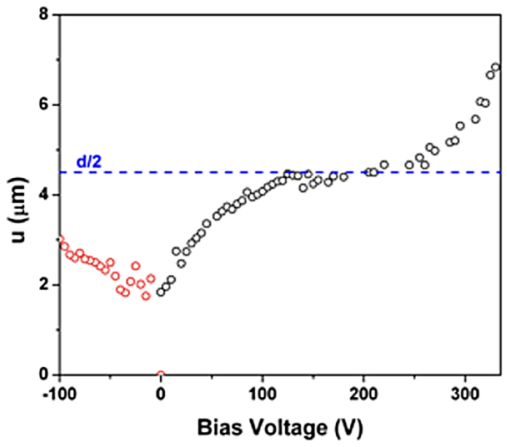

(b)

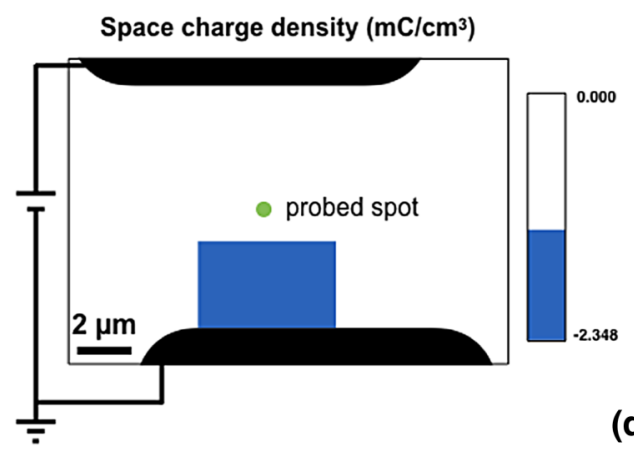

(c)

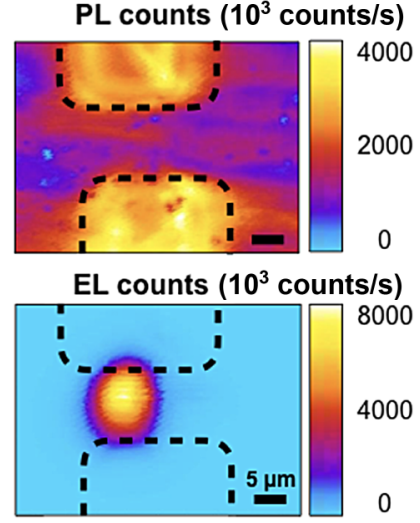

FIG. 5. (a) Extension from the cathode of the space-charge region as a function of the positive (black circles) and negative (red circles) applied bias voltage. (b) Map of the space-charge distribution assumed as the source term for the two-dimensional FEM simulation of the electrostatics of the junction at the bias voltage of $-50 \mathrm{~V}$. The green circle corresponds to the probed spot highlighted in Fig. 3(a). (c) PL map of the junction. The dashed lines highlight the edges of the graphitic electrodes. (d) EL map acquired from the same scan area under an applied bias voltage of $+450 \mathrm{~V}$. 


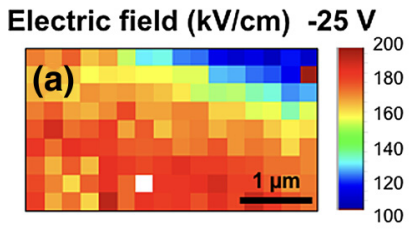

Simulated
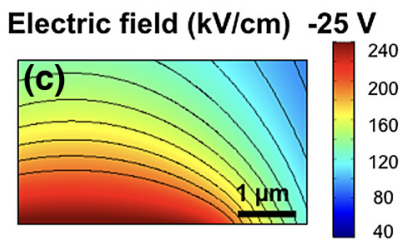

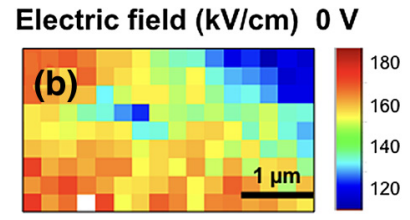

Simulated
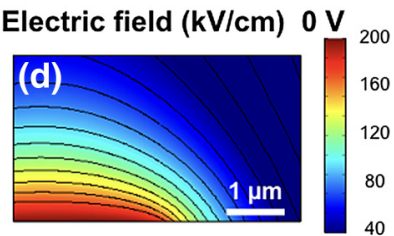

FIG. 6. (a),(b) Electric-field maps from the same regions under -25 and $0 \mathrm{~V}$ applied bias voltage, respectively. A twodimensional FEM simulation of the electric-field amplitude distribution (c) at $V_{\text {bias }}=-25 \mathrm{~V}$ and (d) at $V_{\text {bias }}=0 \mathrm{~V}$ assuming a space-charge extension $u(V)=3 \mu \mathrm{m}$. The black lines represent the isofield lines.

assumed to monotonically increase with the applied bias to progressively fill the active region:

$$
\nabla \cdot \mathbf{E}(x)=\rho(x) /\left(\varepsilon_{r} \varepsilon_{0}\right)=-q N_{T} \theta(u(V)-x) /\left(\varepsilon_{r} \varepsilon_{0}\right)
$$

The value of $N_{T}$ was first determined by matching the value of the simulated electric field with the experimental data, under the constraint that the space-charge extension reaches the probed spot $x=d / 2$ at the bias voltage $V=V_{d / 2}$. This led to an estimation of the trap density of $N_{T}=1.67 \times 10^{16} \mathrm{~cm}^{-3}$, roughly an order of magnitude higher than was determined by a previous electrical characterization of the junction under the assumption of SCLC conduction [40,41]. After the determination of $N_{T}$, by matching the simulated and experimental values of the electric-field amplitude [the dashed blue line in Fig. 4(a) represents the simulated data], it was possible to derive the voltage dependence of the space-charge extension, as shown in Fig. 5(a) (black circles). The curve highlights a significant residual space-charge extension from the unbiased device $[u(V=0) \cong 2 \mu \mathrm{m}]$, which is ascribed to the prior biasing of the junction. Indeed, the permanent polarization due to the formation of persistent regions of high-density space charge upon previous biasing is a wellknown effect in several types of diamond-based devices [16-18,50,51].

\section{Bias polarity reversal}

The same approach was subsequently adopted to investigate the voltage-dependent space-charge buildup at negative bias voltages. The red data points in Fig. 5(a) correspond to the negative-voltage sweep labeled as (3) in Fig. 3(e). Differently from the positive-voltage sweep, the analysis yielded a negligible extension of the space-charge region for the unbiased junction, as supported by the less pronounced increase of the electric amplitude at increasing applied bias. This result indicates that the operation of the junction at $V_{\text {bias }}>+V_{C}$ in the $\mathrm{PF}$ conduction regime in the previous voltage sweep resulted in a reset [label [R] in Fig. $3(\mathrm{e})]$ of the electron traps in the active region. This interpretation is in agreement with the observation of a strong electroluminescent emission at $V_{\text {bias }}>+V_{C}$ from neutral $\mathrm{N}-V^{0}$ centers [22], which is typically interpreted as the consequence of electron-hole-pair recombination at the defect sites $[15,52,53]$. In this context, the concurrent injection of both carrier species in the high-current regime is interpreted as the cause of a progressive trap-neutralization process in the active region.

The $u(V)$ dependence reported in Fig. 5(a) was also exploited to carry a two-dimensional FEM simulation of the horizontal profile of the electric-field amplitude acquired at $-50 \mathrm{~V}$ bias voltage after the $(0-105)-\mathrm{V}$ sweep [label [4] in Fig. 3(e)]. A 5- $\mu \mathrm{m}$ lateral width of the spacecharge distribution [Fig. 5(b)] was assumed to achieve an optimal agreement with the shape of the electric-field map measurements discussed in the following. While a $5-\mu \mathrm{m}$ width could be somewhat counterintuitive, this value was determined by analyzing the extension of the region of the junction that displayed an intense emission in electroluminescence (EL) regime. Indeed, the comparison of confocal maps acquired under PL and EL excitation [Figs. 5(c) and 5(d)] provides a strong indication that the carriers' injection and subsequent trapping is prominently localized in a relatively small section of the junction, where a $\sim 10$ $\mu \mathrm{A}$ current is achieved at $+450 \mathrm{~V}$ applied bias voltage. It is therefore reasonable to expect that the space charge is concentrated in the same volume where the trapping of carriers occurs. The simulated profile is superimposed to the experimental data in Fig. 3(c) (blue line). In consideration of the adopted approximations and assumptions, the simulated profile is in satisfactory agreement with the experimental results, although with a systematic negative offset of $\sim 60 \mathrm{kV} \mathrm{cm}^{-1}$, which is to be ascribed to the formation of a new space-charge extension under increasing device bias at negative voltages [label [C'] in Fig. 3(e)]. The proposed explanation is in line with the observation of a residual space-charge-induced field during the first voltage sweep at increasing positive bias voltages [Fig. 3(d)]. To better appreciate this point, an additional simulation of the electric-field amplitude profile along the horizontal linescan is reported Fig. 3(c) (blue dotted line) under the assumption that the space-charge extension $u$ maintains the maximum value achieved at the end of the negativebias-voltage sweep [configuration [C'] in Fig. 3(e)], i.e., $u=3 \mu \mathrm{m}$. The good agreement of the simulated electricfield strength at the center of the active region indicates that the space charge progressively formed upon an increase of the applied voltage persists when the bias reverts to zero. Furthermore, the profile along the symmetry axis of the junction highlights the smooth variation in the electric 
field across the active region. In this case, the absence of abrupt gradients in the electric-field profile suggests that the peculiar voltage-dependent trend reported in Fig. 3(d) is determined by a high density of carrier traps associated with the flow of a high current density through a $\sim(1 \times 5)-\mu \mathrm{m}^{2}$ section of the junction.

\section{Electric-field mapping}

In order to evaluate the spatial distribution of the electric field in within the active region, ODMR maps were acquired by raster-scanning the sample position over the $5 \times 2 \mu \mathrm{m}^{2}$ area highlighted by the black square in Fig. 3(a). The measurements were carried after the second voltage sweep, i.e., after having polarized the junction at $V_{\text {bias }}=-105 \mathrm{~V}$. To investigate possible polarization effects, a first map at $V_{\text {bias }}=-25 \mathrm{~V}$ [label (5) in Fig. 3(e)] was followed by a second one at $V_{\text {bias }}=0 \mathrm{~V}$ [label (6)].

The local strength of the electric-field amplitude was evaluated on a pixel-by-pixel basis at both bias-voltage configurations according to Eq. (3), following the procedure described in Appendix B. The resulting electric-field maps are displayed in Figs. 6(a) and 6(b), and are to be compared with the result of a FEM simulation [Figs. 6(c) and 6(d)] performed according to the model in Sec. III C [see Fig. 5(b)], where a $u(-25 \mathrm{~V})=u(0 \mathrm{~V})=3 \mu \mathrm{m}$ extension of the space-charge distribution was considered to describe the effects of the residual polarization in the active region of the device.

The agreement between the experimental and simulated electric-field maps is satisfactory, considering the adopted approximations and assumptions. In particular, the electric-field strength displays a significant increase with increasing proximity to the space-charged region. Indeed, the experimental data acquired at $V_{\text {bias }}=-25 \mathrm{~V}$ [Fig. 6(a), median map value $165 \pm 14 \mathrm{kV} \mathrm{cm}^{-1}$ ] are in line with the simulated electric-field strength and distribution [Figs. 6(c) and 6(d)], under the assumption that the same space charge observed during the negativevoltage sweep at a bias of $V=-105 \mathrm{~V}$ [Fig. 5(a)] is still contributing to the electrostatic configuration of the junction.

This effect represents a strong indication that the residual polarization originates from the negative-bias-voltage sweep [labeled as [3] in Fig. 3(e)], which follows the erasing [label (2)] of the internal space charge formed at large positive bias voltages. In a consistent manner, this interpretation is further strengthened by the significant residual electric field observed at $0 \mathrm{~V}$ bias voltage [Fig. 6(b), median map value $153 \pm 11 \mathrm{kV} \mathrm{cm}^{-1}$ ], which supports the initial assumption that a progressive charging of the active region occurs upon each electric bias cycle.

\section{CONCLUSIONS}

We have demonstrated that the ODMR readout from ensembles of $\mathrm{N}-V$ centers allows the investigation of the local effects of charged traps on the internal electric field of a diamond-graphite-diamond junction. This analysis has highlighted the formation of a space-charge distribution and has resulted in the direct observation of memory effects associated with the sample biasing history. This direct experimental insight represents significant progress with respect to conventional approaches, in which complex charge-transport dynamics in defective diamond have been indirectly inferred on the basis of electrical and optical characterizations [20-22].

In particular, the microscale mapping of the internal electric field via ODMR has highlighted the strong persistence of long-lived charged traps in ion-irradiated diamond and provided a new insight into their role in the polarization effects. These phenomena have been extensively studied in the literature only by means of conventional electrical characterization and current-transient detection techniques [16-18,50,51], and have therefore been limited to the macroscopic scale.

The availability of an experimental approach for the direct measurement of the internal electric-field distribution is particularly appealing due to the enticing electronic properties of diamond for emerging applications in radiation-harsh environments, where the understanding of the progressive modification of the internal electric field in the devices' active regions as a function of the lattice damage [7,54-56] is crucial. Furthermore, the availability of the proposed diagnostic method would provide previously unavailable feedback for the design and operation of diamond devices for several technological applications, including beam monitors for high-energy particle experiments [57-59], thin membranes [17,60,61], light-emitting devices [53,62], Schottkybarrier diodes [63,64], and dosimeters for clinical treatments [65].

It is worth remarking that the results presented in this work have relied on the ODMR analysis of the active region of a graphite-diamond-graphite junction under $\mathrm{cw}$ excitation. In view of the significant advances in both field sensitivity and time-resolution achieved by pulsed ODMR schemes $[47,66-68]$ in recent years, we envisage the future possibility of investigating transient fields and assessing the space-charge buildup and reset over time, and concurrently providing direct identification of the trap species based on their charging and discharging dynamics. Furthermore, the future exploitation of advanced ODMR sensing protocols $[10,11,32,56,69,70]$ combined with the use of new fabrication techniques for the creation of graphitic nanochannels [71] opens up interesting perspectives for the investigation of the effects of individual defects $[33,72]$ in diamond, with a potential impact on the development of 
integrated devices for quantum technologies. Finally, the recent discovery of new classes of spin-sensitive defects offering ODMR capabilities in different host materials with appealing properties such as silicon carbide [73] could lead to a wider application of the proposed diagnostic approach to the development of solid-state devices for technological applications.

\section{ACKNOWLEDGMENTS}

We gratefully acknowledge L. Croin (INRiM), S. Brasolin, P. Mereu, B. Pini, and F. Dumitrache (INFNSezione di Torino), and D. Bertoni, N. Dibiase, and O. Giuliano (Physics Department, University of Turin) for their technical support. This research was supported by the following projects: DIESIS project funded by the Italian National Institute of Nuclear Physics (INFN)-CSN5 within the "Young research grant" scheme, Coordinated Research Project "F11020" of the International Atomic Energy Agency (IAEA), the EMPIR17FUN01 "Become" and 17FUN06 "SIQUST" projects (the EMPIR initiative is co-funded by the EU H2020 and the EMPIR Participating States), and the "PiQUET" Project, funded by the Regione Piemonte "Infra-P" program in the framework of EU POR-FESR 2014-2020. J.F. gratefully acknowledges the CERIC-ERIC Consortium for the financial support to the access to the Laboratory for Ion Beam Interactions of the Ruđer Bošković Institute (Proposal No. 20162021). P.O. and F.B. acknowledge support from the project "Departments of Excellence" (L. 232/2016), funded by the Italian Ministry of Education, University and Research (MIUR).

\section{APPENDIX A: ELECTRIC-FIELD SENSING BY THE ODMR TECHNIQUE}

A simplified diagram reporting the energy levels of the $\mathrm{N}-V$ center is shown in Fig. 7(a) [46]. The optical excitation and subsequent radiative relaxation of the $\mathrm{N}-V$ center (with zero-phonon emission at $638 \mathrm{~nm}$ and an additional phonon replica at higher wavelengths) result from the main spin-preserving optical transitions [Fig. 3(a), red arrows] between the ground state $\left({ }^{3} A_{2}\right.$ symmetry) and the ${ }^{3} E$ excited state, which are both spin triplets $(s=1)$ $[43,45,47]$.

An additional decay path [Fig. 7(a), dotted gray arrow] involving a long-lived shelving state (not shown in the figure) is available for the energy relaxation at a significantly higher rate for the excited $s= \pm 1$ spin states with respect to the $s=0$ one. This non-spin-preserving path occurs through a nonradiative transition and results in the relaxation of the system into the $s=0$ state of the ${ }^{3} A_{2}$ ground level. As the lifetime of the shelving state (250 ns [45]) is significantly higher than that of the direct spin-preserving optical transition ( $\sim 12$ ns [48]), a $\sim 30 \%$ contrast in the photon-emission intensity exists between the $s= \pm 1$ and the $s=0$ spin states, which can be exploited to perform an optical readout of the N- $V$ center's spin state [45].

Indeed, the degeneracy between the $s=0$ and the $s= \pm 1$ states is lifted by the fine-structure energy splitting $h D$ for the ground state [see Fig. A1(a), green arrow], taking into account the electronic spin-spin interaction $D_{0} \sim 2.87 \mathrm{GHz}$ (zero-field splitting in the following) plus additional contributions due to interactions with external fields [43-45]. Specifically, the ODMR technique relies on the measurement of the luminescence intensity as a function of the frequency of a concurrent microwave excitation. This procedure enables the identification of the energy resonances corresponding to the $s=0 \rightarrow s= \pm 1$ transitions, which are in turn accompanied by a luminescence intensity decrease [Fig. A1(b)], thus providing a measurement of the finestructure shifts and of the sublevel splittings [Fig. A1(a), blue arrow] associated with the interaction of the spin states with external fields [43,44,46,49].

The Hamiltonian of the ground state of the N- $V$ system, describing the energy levels of the electronic spin states due to the spin $(S)$ interaction with the static magnetic $(B)$, electric $(E)$, and strain $(F)$ fields, can be written in terms of the natural spin-triplet basis $\left\{\left|m_{s}=0\right\rangle,\left|m_{s}=+1\right\rangle\right.$, $\left.\left|m_{s}=-1\right\rangle\right\}$ in the following matrix form $[32,43]$ :

$$
\frac{\hat{H}_{s}}{h}=\left(\begin{array}{ccc}
0 & -\mu g \frac{B_{x}-i B_{y}}{\sqrt{2}} & -\mu g \frac{B_{x}+i B_{y}}{\sqrt{2}} \\
-\mu g \frac{B_{x}+i B_{y}}{\sqrt{2}} & D+\mu g B_{\|} & -d_{\perp}\left(P_{x}-i P_{y}\right) \\
-\mu g \frac{B_{x}-i B_{y}}{\sqrt{2}} & -d_{\perp}\left(P_{x}+i P_{y}\right) & D-\mu g B_{\|}
\end{array}\right),
$$

where $D=D_{0}+d_{\|} P_{\|}$describes the frequency shift of the resonance lines resulting from the zero-field splitting and from the Stark effect associated with the component of the $\mathbf{P}=\mathbf{E}+\mathbf{F}$ vector parallel to the N- $V$ axis, with coupling constant $d_{\|}=0.35 \mathrm{~Hz} \mathrm{~V}^{-1}$ [32]. The $\mu g B_{\|}$term on the matrix diagonal describes the Zeeman splitting of the ODMR resonances ( $\mu$ is the Bohr magneton and $g$ is the ground-state electronic $g$ factor [43]), and indicates that the natural-spin basis vectors are eigenstates of the Hamiltonian in the sole presence of the magnetic field aligned with the $\mathrm{N}-V$ axis.

The presence of additional transverse (i.e., lying in the $x-y$ plane in the current notation) strain- and electric-field components $P_{\perp}$ (with coupling constant $d_{\perp}=17 \mathrm{~Hz} \mathrm{~V}^{-1} \mathrm{~cm}$ [32]) modifies the ground-state hyperfine structure. The Hamiltonian assumes a quasidiagonal form considering a new spin basis $\{|0\rangle,|+\rangle,|-\rangle\}$, obtained by a field-dependent mixing of the $|+1\rangle$ and $|-1\rangle$ spin 
(a)

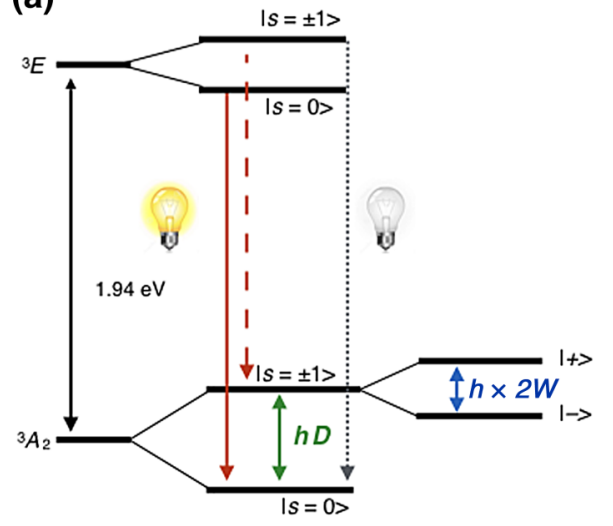

(b)

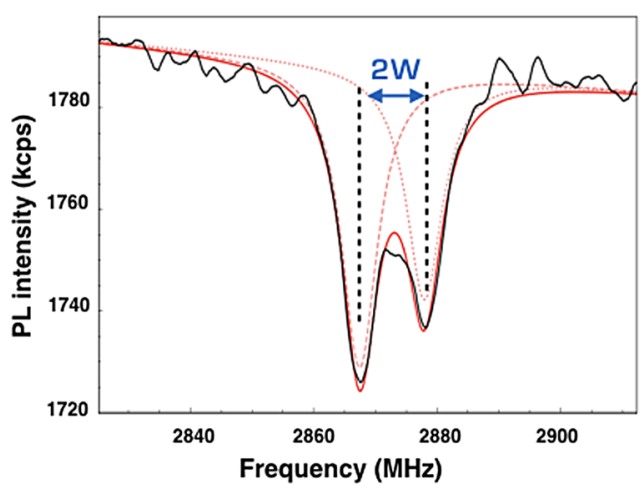

FIG. 7. (a) Schematic representation of the relevant energy levels of the negatively charged $\mathrm{N}-\mathrm{V}$ center. (b) typical ODMR spectrum acquired at $+25 \mathrm{~V}$ bias voltage (black line). The fitting curve (solid red) is superimposed, together with the two corresponding Lorentzian peaks. states [44] according to the following unitary operator:

$$
\hat{U}=\left(\begin{array}{ccc}
1 & 0 & 0 \\
0 & e^{i \phi / 2} \sin \left(\frac{\theta}{2}\right) & e^{-i \phi / 2} \cos \left(\frac{\theta}{2}\right) \\
0 & e^{i \phi / 2} \cos \left(\frac{\theta}{2}\right) & -e^{-i \phi / 2} \sin \left(\frac{\theta}{2}\right)
\end{array}\right),
$$

where $\tan (\varphi)=P_{\mathrm{y}} / P_{\mathrm{x}}$ and $\tan (\theta)=d_{\perp} P_{\perp} /\left(\mu g B_{\|}\right)$are the field-dependent phases defining the spin state mixing [44]. With further consideration of the quantity $W$,

$$
W=\left[\left(\mu g B_{\|}\right)^{2}+\left(d_{\perp} P_{\perp}\right)^{2}\right]^{1 / 2},
$$

defined by imposing $\mu g B_{\|}=W \cos (\theta), d_{\perp} P_{\perp}=W \sin (\theta)$, the Hamiltonian in Eq. (A1) takes the following form in the $\{|0\rangle,|+\rangle,|-\rangle\}$ basis:

$$
\frac{\hat{H}_{d}}{h}=\frac{1}{h} \hat{U} \hat{H}_{s} \hat{U}^{\dagger}=\left(\begin{array}{ccc}
0 & c_{1} \mu g B_{\perp} & c_{2} \mu g B_{\perp} \\
c_{1}^{*} \mu g B_{\perp} & D+W & 0 \\
c_{2}^{*} \mu g B_{\perp} & 0 & D-W
\end{array}\right),
$$

\section{$\mathrm{D}(\mathrm{MHz}) \mathrm{OV}$}

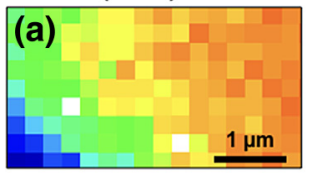

$\mathrm{D}(-25 \mathrm{~V})$ - $\mathrm{D}(\mathrm{OV})(\mathrm{MHz})$

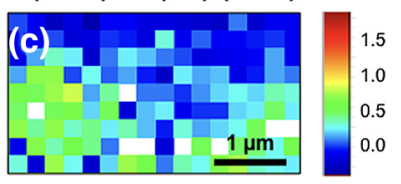

D (MHz) $-25 \mathrm{~V}$
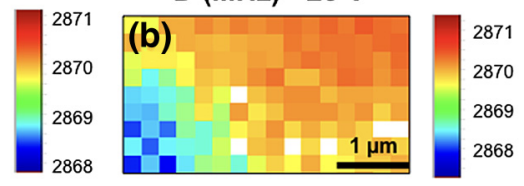

Stress (GPa)

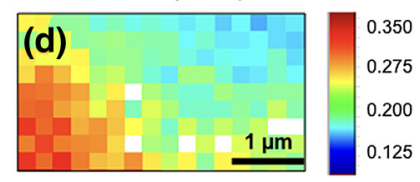

FIG. 8. (a),(b) Maps of the $D$ parameter from the $(5 \times 2)-\mu \mathrm{m}^{2}$ region highlighted in Fig. 3(a), as evaluated from the ODMR spectra on a pixel-by-pixel basis, under an applied bias voltage of -25 and $0 \mathrm{~V}$, respectively. (c) Difference in the $D$ value between the maps acquired at -25 and $0 \mathrm{~V}$. (d) Corresponding value of the local stress evaluated from the map acquired at $-25 \mathrm{~V}$ applied bias. where $c_{1}$ and $c_{2}$ describe the relative phase of the matrix elements and $B_{\perp}$ is the transverse component of the magnetic field with respect to the axis of the $\mathrm{N}-V$ center. If an ensemble of $\mathrm{N}-V$ centers, distributed according to all the four possible orientations in the diamond lattice, is considered, we can assume that $B_{\perp} \sim B_{\|}$and $B_{\perp}^{2} /(D W) \ll 1$, i.e., the nondiagonal terms can be neglected and the Hamiltonian in Eq. (A3) can be regarded as diagonal in the basis $\{|0\rangle,|+\rangle,|-\rangle\}[44]$. It is worth noting that, since only the $m_{\mathrm{s}}= \pm 1$ states are involved in the spin-state mixing of $|+\rangle$ and $|-\rangle$ in Eq. (A2), their resonant microwave-induced transitions with the $|0\rangle$ state still result in a detectable PL contrast, thus ensuring the suitability of the ODMR technique for electric- and strain-field sensing. Therefore, the energy difference between the between the $\mid 0>$ and the

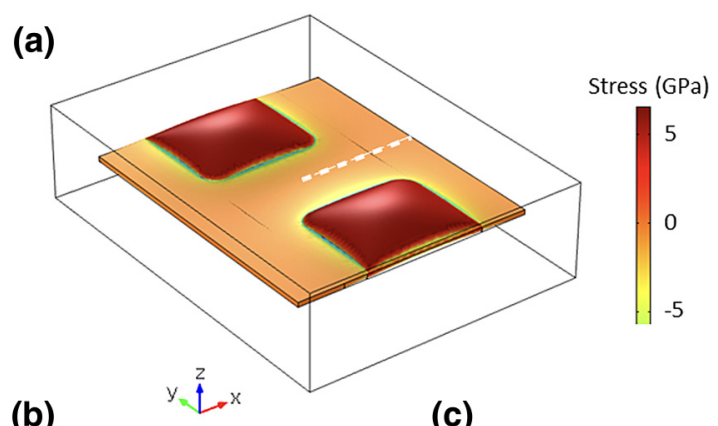

(b)

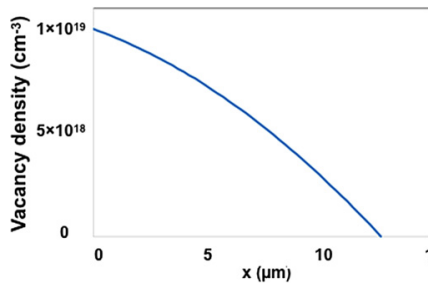

(c)
FIG. 9. (a) Color map of the volumetric stresses in the region under study, due to volume expansion of the ion-implanted areas.

(b) Vacancy-density distribution along the dotted line in (a).

(c) Corresponding stress distribution (dashed blue line) to be compared with experimental results deriving from the ODMR measurements (red dots). 
$\mid \pm>$ states is given by $h(D \pm W)$, and the observed ODMR resonances are separated by a $2 W$ frequency splitting [Fig. 7(b)], which is determined by the strengths of the magnetic $(B)$, electric $(E)$, and strain $(F)$ fields, as well as their orientations with respect to the axes of the $\mathrm{N}-V$ center, according to Eq. (A3).

\section{APPENDIX B: ANALYSIS OF THE ODMR MEASUREMENTS}

The transverse component of the electric field was derived from the ODMR spectra through Eq. (A3) as

$$
d_{\perp} E_{\perp}=-k_{\perp} \Sigma+\left[W^{2}-\left(\mu g B_{\|}\right)^{2}\right]^{1 / 2} .
$$

This operation relied on preliminary knowledge of the $B_{\|}$and $\Sigma$ fields, whose analysis and quantification are discussed in detail in the following subsections.

\section{Magnetic field contribution}

The coupling of the $\mathrm{N}-V$ center spin with the environmental magnetic field results in the Zeeman splitting term $W_{1}=\mu_{B} g B_{\|}$, which does not contribute to the resonance shift $D$ [see Eqs. (A1)-(A3)]. The resonance splitting was evaluated from the ODMR spectrum acquired at $0 \mathrm{~V}$ bias before the voltage sweep at negative polarity [label [R] in Fig. 3(e)], from the probed spot at the center of the active region [green spot in Fig. 3(a)]. In the absence of the external electric field, the relevant energy-splitting term reduces to the sole contribution of the strain vector field $\mathbf{P}=\mathbf{F}$, and Eq. (B1) reduces to $\mu g B_{\|}=\left[(W)^{2}-\right.$ $\left.\left(d_{\perp} F_{\perp}\right)^{2}\right]^{1 / 2}$. A $0.46-\mathrm{MHz}$ increment in the zero-field splitting term $W$ associated with the local radiation-induced strain field (corresponding to a $\sim 0.24 \mathrm{GPa}$ stress along the $\langle 100\rangle$ crystallographic axis) was derived, as discussed in more detail in the next subsection, leading to an estimation of the $\mu g B_{\|}$value of $1.09 \mathrm{MHz}$, which in turn corresponds to a $39 \mu \mathrm{T}$ magnetic field. Due to the absence of a magnetic screening of the experimental setup, this value is ascribed to the earth and environmental magnetic field and is assumed to be constant in the whole region investigated in this work. This assumption is supported by the fact that any ferromagnetic component of the confocal microscopy setup was left at a fixed position for the whole duration of the measurements.

It is also worth noting that the magnetic field associated with the current flowing in the active region of the device is negligible, as it is well below the sensitivity of the experimental setup operating in $\mathrm{cw}$ mode. Indeed, even under the unrealistic assumption that a current of $10 \mu \mathrm{A}$ (i.e., at $V=V_{C}$; see Fig. 2) is entirely flowing within a $1-\mu \mathrm{m}$ distance (i.e., the focal point size) from the sensed position, the induced magnetic field would be $<200 \mathrm{nT}$, according to the Biot-Savart law [33].

\section{Evaluation of the internal stress}

The mechanical stresses associated with the presence of radiation-induced lattice damage [74] within the active region of the junction are included in Eqs. (1)-(4) in the effective electric-field vector $\mathbf{P}$. The component of such a vector parallel to the $\mathrm{N}-V$ center axis (i.e., $P_{\|}$) induces a shift in the ODMR resonances. This term can be rewritten as $d_{\|} P_{\|}=d_{\|}\left(F_{\|}+E_{\|}\right)=k_{\|} \Sigma+d_{\| \mid} E_{\|}$, where $\Sigma$ is the strength of the stress field associated with the parallel lattice strain and $k_{\|}=14.6 \mathrm{MHz} \mathrm{GPa}^{-1}$ is the experimental value of the coupling constant along the $\mathrm{N}-V$ axis [46]. The electric-field coupling constant $d_{\|}=0.35 \mathrm{~Hz} \mathrm{~cm} \mathrm{~V}^{-1}$ is negligible with respect to $k_{\|}$, resulting in the approximated expression $D \cong D_{0}+k_{\|} \Sigma$.

In order to experimentally assess the value of $D_{0}$ (i.e., the $D$ value in absence of mechanical stresses), we estimated the $D$ value from the ODMR spectrum acquired corresponding to the largest distance probed from the central axis of the active region, i.e., $x_{0} \cong 12 \mu \mathrm{m}$ across the linescan reported in Figs. 3(a) and 3(c). Indeed, according to the results of three-dimensional FEM simulations of the stress-field strength [75] in the same geometry [Fig. 4(a)], such a distance is sufficient to probe a region with a negligible stress field. The zero-field splitting was therefore evaluated as $D_{0}=D\left(x=x_{0}\right)=(2872.9 \pm 0.2) \mathrm{MHz}$. The local stress-induced shift was then derived for the whole linescan as $\Sigma(x)=\left[D_{0}-D(x)\right] / k_{\|}$, and resulted in a $<0.25$ GPa tensile stress at the central position of the active region [Figs. 3(c) and 8(d)].

The mechanical stresses building up in the active region are due to two effects, i.e., (i) the constrained volume expansion of the graphitic microelectrodes with respect to the surrounding diamond matrix, and (ii) the volumetric expansion of the defective region surrounding the same microelectrodes. These two mechanisms induce, respectively, a compressive and a tensile stress in the active region of the device, and their concurrent effect was numerically modeled by performing three-dimensional finiteelement simulations, as described in Ref. [75]. The simulated volume is shown in Fig. 7. The following mechanical parameters were adopted for diamond and amorphous carbon, respectively: mass densities $\rho_{d}=3515 \mathrm{~kg} \mathrm{~m}^{-3}$ and $\rho_{\mathrm{aC}}=2060 \mathrm{~kg} \mathrm{~m}^{-3}$, Young's moduli $E_{d}=1220 \mathrm{GPa}$ and $E_{\mathrm{aC}}=21.38 \mathrm{GPa}[76,77]$. The defective diamond region between the electrodes shown in Fig. 1(d) is assumed, in a first approximation, to contain a vacancy density of $\lambda=10^{19} \mathrm{~cm}^{-3}$ (i.e., a reduction of three orders of magnitudes with respect to the graphitization threshold) due to irradiation with stray ions, as well as vacancy diffusion resulting from an incomplete recovery of the crystal structure after annealing. The vacancy density decreases along the $x$ direction, until it becomes negligible at a significant (i.e., $\sim 10 \mu \mathrm{m}$, corresponding to roughly twice the size of the ion microbeam) distance from the nominally irradiated region where the graphitic electrodes are formed. The local 
mass density and Young's modulus of the defective region of interest can thus be described by a semiempirical model taking into account damage saturation effects [75]:

$$
\rho(x)=\rho_{d}-\left(\rho_{d}-\rho_{\mathrm{aC}}\right)\left(1-\exp \left[-\frac{\lambda(x)}{\gamma\left(1-\rho_{d} / \rho_{\mathrm{aC}}\right)}\right]\right),
$$

where $\gamma=1.77 \times 10^{23} \mathrm{~cm}^{-3}$ is the atomic density of diamond, $\lambda(x)$ is the vacancy density, and

$$
E(x)=E_{d}\left(1-\kappa\left[-\frac{\rho(x)}{\rho_{d}}\right]\right),
$$

where $\kappa$ is an empirical constant [75]. The density decrease due to irradiation generates a constrained expansion of the implanted volume, which is only partially counterbalanced by the expansion of the surrounding graphitic electrodes, resulting in residual strains in the $i=x, y, z$ directions given by

$$
\varepsilon_{i}(x)=\sqrt[3]{\frac{\rho_{d}}{\rho(x)}}-1 .
$$

Figure 7(a) shows a color-encoded map of the corresponding FEM-calculated internal volumetric stress $\sigma_{V}=\left(\sigma_{x}^{2}+\sigma_{z}^{2}+\sigma_{z}^{2}\right)^{1 / 2}$ in the material. The stresses reach relatively high compressive values ( $\sim 5 \mathrm{GPa}$ ) close (i.e., at $<1 \mu \mathrm{m}$ ) to the electrodes, where the largest density mismatch occurs, and smaller $(<1 \mathrm{GPa})$ tensile values at the center of the active region of the device. The estimated vacancy-density distribution along the dotted white line in Fig. 9(a) is shown in Fig. 9(b), and the corresponding stresses are shown in Fig. 9(c) (the curve labeled as FEM). In particular, the calculated tensile stresses along the dotted line are in satisfactory agreement (at least from an orderof-magnitude point of view) with those that were experimentally derived from the $D(x)$ frequency shift along the linescan reported in Fig. 3(c) (Exp in the plot), confirming the validity of the assumed vacancy distribution.

The contribution of the stress field to the resonance splitting was then experimentally determined as $d_{\perp} F_{\perp}(x)=k_{\perp} \Sigma(x)$, as described in the following. The coupling constant $k_{\perp}=1.93 \mathrm{MHz} \mathrm{GPa}^{-1}$ was derived from the experimental data reported in Ref. [46], where the ODMR splitting as a function of the applied stress field is evaluated in a sample that has the same crystallographic orientation as the substrate investigated in this work. The fitting of the data reported in Ref. [46] (Fig. 3, 0.01-0.5GPa range) was performed after suitable removal of the environmental magnetic field (which was assumed to cause the resonance splitting at the minimum stress value of $0.1 \mathrm{MPa}$ ), according to Eq. (A3). Such estimation of the coupling constant $k_{\perp}$ enabled the local evaluation of the stress contribution to $W$, while the stress field $\Sigma(x)$ was determined by the measured resonance shift $D(x)$. Therefore, it was possible to subtract this contribution from the experimental ODMR data according to Eq. (B1), as well as to identify the background magnetic field at the central position of the junction, having set $E_{\perp}=0$ [see Appendix $\mathrm{B}(1)]$.

\section{Temperature effects}

Temperature variations affect the fine structure of the $\mathrm{N}-V$ center through the thermal expansion of the lattice constant, resulting in the creation of an additional strain field besides that associated with induced structural damage [49]. While the ODMR experiments were performed at room temperature, the Joule heating associated with the current flow in the active region could, in principle, induce a local temperature increase. To test this hypothesis, the $D$ - $D_{0}$ shift (i.e., the contribution to the resonances shift resulting from mechanical stresses) was investigated in the ODMR spectra acquired during the $0-350-\mathrm{V}$ voltage sweep reported in Fig. 3(b). Since this data set was acquired at a fixed position, any voltage-dependent trend in the ODMR resonances shift would not be affected by damage-induced stress contributions. None of the $D$ values displayed any significant dependence on the applied bias voltage, i.e., on the current injected into the junction, since they ranged within $\sim 2 \mathrm{MHz}$ around the $2872.1-\mathrm{GHz}$ value. This variability imposes an upper limit on the temperature variation of $\sim 30 \mathrm{~K}[49]$.

\section{ODMR mapping of internal stresses}

Figures 8(a) and 8(b) display the maps of the zero-field splitting $D$ evaluated from the ODMR resonances under an external applied bias voltage of 0 and $-25 \mathrm{~V}$, respectively. The difference between the zero-field splitting values $D$ acquired in the two maps shown in Fig. 8(c) indicates values close to zero within the limits determined by the $\sim 2-\mathrm{MHz}$ FWHM of the ODMR dips. As the shift is sensitive only to electric and stress fields [see Eq. (A1)], the observation that the $D$ parameter is insensitive to changes in the applied bias voltages indicates that the spatial distribution of its shift values is primarily determined by the internal stress fields. Thus, the same procedure adopted for the analysis of the voltage-dependent ODMR spectra discussed in Secs. III B and III C and in Appendixes B 1-B 3 allowed the mapping of the internal tensile stresses [Fig. $8(d)$ ], with typical values in the $0.2-0.3$-GPa range, in satisfactory agreement with the results obtained from FEM simulations of the mechanical deformations of the sample [see Appendix B(2)].

\section{APPENDIX C: DEVICE-FABRICATION DETAILS}

\section{Ion-beam fabrication of graphitic electrodes}

The electrodes were fabricated in a planar geometry using a $6-\mathrm{MeV} \mathrm{C}^{3+}$ beam. An ion fluence of 
$\sim 4 \times 10^{16} \mathrm{~cm}^{-2}$ was chosen to overcome the graphitization threshold of diamond $\left[(6-9) \times 10^{22} \mathrm{~cm}^{-3}\right.$ for deep $\mathrm{MeV}$ ion implantation [37,38]]. The amorphized layer at the end of the ion range was converted to a graphitic phase by a subsequent thermal annealing performed at $950^{\circ} \mathrm{C}$ for $2 \mathrm{~h}$ (pressure $<6 \times 10^{-4} \mathrm{~Pa}$ ).

\section{PL and ODMR measurements}

Measurements were performed using a confocal microscope [15] with 515-nm laser excitation. The luminescent emission reported in the PL maps and ODMR spectra was acquired in the $650-800-\mathrm{nm}$ spectral range. CW microwave excitation was sourced by a $\mathrm{Cu}$ wire $(\emptyset \sim 30$ $\mu \mathrm{m})$ placed on the sample surface at a distance of $\sim 25 \mu \mathrm{m}$ from the active region. The acquisition of ODMR spectra required a $\sim 5$-min acquisition time at each individual point. Therefore, the experimental data discussed in this work are limited to the stationary electric-field distribution resulting from the charging of long-lived carrier traps.

[1] R. Nemanich, J. Carlisle, J. A. Hirata, and K. Haenen, CVD diamond - research, applications, and challenges, MRS Bull. 39, 490, (2014).

[2] C. E. Nebel, Electronic properties of CVD diamond, Semicond. Sci. Technol. 18, S1 (2003).

[3] J. W. Liu, M. Y. Liao, M. Imura, E. Watanabe, H. Oosato, and Y. Koide, Diamond logic inverter with enhancementmode metal-insulator-semiconductor field effect transistor, Appl. Phys. Lett. 105, 082110 (2014).

[4] C. Verona, W. Ciccognani, S. Colangeli, F. Di Pietrantonio, E. Giovine, E. Limiti, M. Marinelli, and G. Verona-Rinati, Gate-source distance scaling effects in H-terminated diamond MESFETs, IEEE Trans. Electron Devices 62, 1150 (2015).

[5] J. Seco, B. Clasie, and M. Partridge, Review on the characteristics of radiation detectors for dosimetry and imaging, Phys. Med. Biol. 59, R303 (2014).

[6] M. Scaringella, M. Zani, A. Baldi, M. Bucciolini, E. Pace, A. De Sio, C. Talamonti, and M. Bruzzi, First dose-map measured with a polycrystalline diamond 2D dosimeter under an intensity modulated radiotherapy beam, Nucl. Instr. Methods Phys. Res. A 796, 89 (2015).

[7] S. Lagomarsino, M. Bellini, C. Corsi, V. Cindro, K. Kanxheri, A. Morozzi, D. Passeri, L. Servoli, and C. J. Schmidt, Radiation hardness of three-dimensional polycrystalline diamond detectors, Appl. Phys. Lett. 106, 193509 (2015).

[8] J. B. S. Abraham, B. A. Aguirre, J. L. Pacheco, G. Vizkelethy, and E. Bielejec, Fabrication and characterization of a co-planar detector in diamond for low energy single ion implantation, Appl. Phys. Lett. 109, 063502 (2016).

[9] A. Bendali, C. Agnès, S. Meffert, V. Forster, A. Bongrain, J.-C. Arnault, J.-A. Sahel, A. Offenhäusser, P. Bergonzo, and S. Picaud, Distinctive glial and neuronal interfacing on nanocrystalline diamond. PLoS ONE 9, e92562 (2014).

[10] F. Picollo, A. Battiato, E. Carbone, L. Croin, E. Enrico, J. Forneris, S. Gosso, P. Olivero, A. Pasquarelli, and V.
Carabelli, Development and characterization of a diamondinsulated graphitic multi electrode array realized with ion beam lithography, Sensors 15, 515 (2015).

[11] M. Gulka, E. Bourgeois, J. Hruby, P. Siyushev, G. Wachter, F. Aumayr, P. R. Hemmer, A. Gali, F. Jelezko, M. Trupke, and M. Nesladek, Pulsed Photoelectric Coherent Manipulation and Detection of N- $V$ Center Spins in Diamond, Phys. Rev. Appl. 7, 044032 (2017).

[12] Y. Doi, T. Makino, H. Kato, D. Takeuchi, M. Ogura, H. Okushi, H. Morishita, T. Tashima, S. Miwa, S. Yamasaki, P. Neumann, J. Wrachtrup, Y. Suzuki, and N. Mizuochi, Deterministic Electrical Charge-State Initialization of Single Nitrogen-Vacancy Center in Diamond, Phys. Rev. X 4, 011057 (2014).

[13] L. C. Bassett, F. J. Heremans, C. G. Yale, B. B. Buckley, and D. D. Awschalom, Electrical Tuning of Single Nitrogen-Vacancy Center Optical Transitions Enhanced by Photoinduced Fields, Phys. Rev. Lett. 107, 266403 (2011).

[14] A. Lohrmann, S. Pezzagna, I. Dobrinets, P. Spinicelli, V. Jacques, J.-F. Roch, J. Meijer, and A. M. Zaitsev, Diamond based light-emitting diode for visible single-photon emission at room temperature, Appl. Phys. Lett. 99, 251106 (2011).

[15] J. Forneris, P. Traina, D. Gatto Monticone, G. Amato, L. Boarino, G. Brida, I. P. Degiovanni, E. Enrico, E. Moreva, V. Grilj, N. Skukan, M. Jakšić, M. Genovese, and P. Olivero, Electrical stimulation of non-classical photon emission from diamond color centers by means of sub-superficial graphitic electrodes, Sci. Rep. 5, 15901 (2015).

[16] C. Manfredotti, A. Lo Giudice, E. Vittone, F. Fizzotti, Y. Garino, and E. Pace, Memory effects in CVD diamond, Diam. Relat. Mater. 15, 1467 (2006).

[17] W. Kada, N. Iwamoto, T. Satoh, S. Onoda, V. Grilj, N. Skukan, M. Koka, T. Ohshima, M. Jakšić, and T. Kamiya, Continuous observation of polarization effects in thin SCCVD diamond detector designed for heavy ion microbeam measurement, Nucl. Instr. Methods Phys. Res. B 331, 113 (2014).

[18] A. Lohstroh, P. J. Sellin, S. G. Wang, A. W. Davies, and J. M. Parkin, Mapping of polarization and detrapping effects in synthetic single crystal chemical vapor deposited diamond by ion beam induced charge imaging, J. Appl. Phys. 101, 063711 (2007).

[19] C. Manfredotti, F. Wang, P. Polesello, E. Vittone, and F. Fizzotti, Blue-violet electroluminescence and photocurrent spectra from polycrystalline chemical vapor deposited diamond film, Appl. Phys. Lett. 67, 3376 (1995).

[20] J. Isberg, J. Hammersberg, E. Johansson, T. Wikström, D. J. Twitchen, A. J. Whitehead, S. E. Coe, and G. A. Scarsbrook, High carrier mobility in single-crystal plasmadeposited diamond, Science 297, 1670 (2002).

[21] P. Gonon, Y. Boiko, S. Prawer, and D. Jamieson, PooleFrenkel conduction in polycrystalline diamond, J. Appl. Phys. 79, 3778 (1996).

[22] J. Forneris, S. Ditalia Tchernij, A. Tengattini, E. Enrico, V. Grilj, N. Skukan, G. Amato, L. Boarino, M. Jakšić, and P. Olivero, Electrical control of deep NV centers in diamond by means of sub-superficial graphitic micro-electrodes, Carbon 113, 76 (2017).

[23] P. Olivero, C. Manfredotti, E. Vittone, F. Fizzotti, C. Paolini, A. Lo Giudice, R. Barrett, and R. Tucoulou, Investigation of chemical vapour deposition diamond 
detectors by X-ray micro-beam induced current and $\mathrm{X}$-ray micro-beam induced luminescence techniques, Spectrochim. Acta Part B 59, 1565 (2004).

[24] E. Vittone, Semiconductor characterization by scanning ion beam induced charge (IBIC) microscopy, ISRN Mater. Sci. 2013, 637608 (2013).

[25] T. Sekiguchi, S. Koizumi, and T. Taniguchi, Characterization of $\mathrm{p}-\mathrm{n}$ junctions of diamond and $\mathrm{c}-\mathrm{BN}$ by cathodoluminescence and electron-beam-induced current, J. Phys.: Condens Matter 16, S91 (2004).

[26] S. Konoa, T. Teraji, H. Kodama, and A. Sawabea, Imaging of diamond defect sites by electron-beam-induced current, Diam. Relat. Mater. 59, 54 (2015).

[27] J. M. Taylor, P. Cappellaro, L. Childress, L. Jiang, D. Budker, P. R. Hemmer, A. Yacoby, R. Walsworth, and M. D. Lukin, High-sensitivity diamond magnetometer with nanoscale resolution, Nat. Phys. 4, 810 (2008).

[28] J. R. Maze, P. L. Stanwix, J. S. Hodges, S. Hong, J. M. Taylor, P. Cappellaro, L. Jiang, M. V. G. Dutt, E. Togan, A. S. Zibrov, A. Yacoby, R. L. Walsworth, and M. D. Lukin, Nanoscale magnetic sensing with an individual electronic spin in diamond, Nature 455, 644 (2008).

[29] V. M. Acosta, E. Bauch, M. P. Ledbetter, A. Waxman, L.-S. Bouchard, and D. Budker, Temperature Dependence of the Nitrogen-Vacancy Magnetic Resonance in Diamond, Phys. Rev. Lett. 104, 070801 (2010).

[30] E. H. Chen, H. A. Clevenson, K. A. Johnson, L. M. Pham, D. R. Englund, P. R. Hemmer, and D. A. Braje, High-sensitivity spin-based electrometry with an ensemble of nitrogen-vacancy centers in diamond, Phys. Rev. A 95, 053417 (2017).

[31] M. W. Doherty, J. Michl, F. Dolde, I, Jakobi, P. Neumann, N. M. Manson, and J. Wrachtrup, Measuring the defect structure orientation of a single centre in diamond, New J. Phys. 16, 063067 (2014).

[32] F. Dolde, H. Fedder, M. W. Doherty, T. Nöbauer, F. Rempp, G. Balasubramanian, T. Wolf, F. Reinhard, L. C. L. Hollenberg, F. Jelezko, and J. Wrachtrup , Electricfield sensing using single diamond spins, Nat. Phys. 7, 459 (2011).

[33] M. Lesik, N. Raatz, A. Tallaire, P. Spinicelli, J. Rogers, J. Achard, A. Gicquel, V. Jacques, J.-F. Roch, J. Meijer, and S. Pezzagna, Production of bulk NV centre arrays by shallow implantation and diamond CVD overgrowth, Phys. Status Solidi A 213, 2594 (2016).

[34] T. Iwasaki, W. Naruki, K. Tahara, T. Makino, H. Kato, M. Ogura, D. Takeuchi, S. Yamasaki, and M. Hatano, Direct nanoscale sensing of the internal electric field in operating semiconductor devices using single electron spins, ACS Nano 11, 1238 (2017).

[35] S. Ditalia Tchernij, N. Skukan, F. Picollo, A. Battiato, V. Grilj, G. Amato, L. Boarino, E. Enrico, M. Jakšić, P. Olivero, and J. Forneris, Electrical characterization of a graphite-diamond-graphite junction fabricated by $\mathrm{MeV}$ carbon implantation, Diam. Relat. Mater. 74, 125 (2017).

[36] J. F. Ziegler, J. P. Biersack, and U. Littmark, The Stopping and Range of Ions in Solids (Pergamon, New York, 2003).
[37] D. P. Hickey, K. S. Jones, and R. G. Elliman, Amorphization and graphitization of single-crystal diamond - A transmission electron microscopy study, Diam. Relat. Mater. 18, 1353 (2009).

[38] T. Lühmann, R. Wunderlich, R. Schmidt-Grund, J. BarzolaQuiquia, P. Esquinazi, M. Grundmann, and J. Meijer, Investigation of the graphitization process of ion-beam irradiated diamond using ellipsometry, Raman spectroscopy and electrical transport measurements, Carbon 121, 512 (2017).

[39] C. Santori, P. E. Barclay, K.-M. C. Fu, and T. G. Beausoleil, Vertical distribution of nitrogen-vacancy centers in diamond formed by ion implantation and annealing, Phys. Rev. B 79, 125313 (2009).

[40] F.-C. Chiu, A review on conduction mechanisms in dielectric films, Adv. Mat. Sci. Eng. 2014, 578168 (2014).

[41] M. A. Lampert, Simplified theory of space-charge-limited currents in an insulator with traps, Phys. Rev. 103, 1648 (1956).

[42] P. Olivero, J. Forneris, M. Jakšić, Ž. Pastuović, F. Picollo, N. Skukan, and E. Vittone, Focused ion beam fabrication and IBIC characterization of a diamond detector with buried electrodes, Nucl. Instr. Methods Phys. Res. B 269, 2340 (2011).

[43] M. W. Doherty, N. B. Manson, P. Delaney, F. Jelezko, J. Wrachtrup, and L. C. L. Hollenberg, The nitrogenvacancy colour centre in diamond, Phys. Rep. 528, 1 (2013)

[44] M. W. Doherty, F. Dolde, H. Fedder, F. Jelezko, J. Wrachtrup, N. B. Manson, and L. C. L. Hollenberg, Theory of the ground-state spin of the NV-center in diamond, Phys. Rev. B 85, 205203 (2012).

[45] R. Schirhagl, K. Chang, M. Loretz, and C. L. Degen, Nitrogen-vacancy centers in diamond: Nanoscale sensors for physics and biology, Annu. Rev. Phys. Chem. 65, 83 (2014).

[46] M. W. Doherty, V. V. Struzhkin, D. A. Simpson, L. P. McGuinness, Y. Meng, A. Stacey, T. J. Karle, R. J. Hemley, N. B. Manson, L. C. L. Hollenberg, and S. Prawer, Electronic Properties and Metrology Applications of the Diamond NV-Center under Pressure, Phys. Rev. Lett. 112, 047601 (2014).

[47] E. Bernardi, R. Nelz, S. Sonusen, and E. Neu, Nanoscale sensing using point defects in single-crystal diamond: Recent progress on nitrogen vacancy center-based sensors, Crystals 7, 124 (2017).

[48] J. Storteboom, P. Dolan, S. Castelletto, X. Li, and M. Gu, Lifetime investigation of single nitrogen vacancy centres in nanodiamonds, Opt. Express 23, 11327 (2015).

[49] M. W. Doherty, V. M. Acosta, A. Jarmola, M. S. J. Barson, N. B. Manson, D. Budker, and L. C. L. Hollenberg, The temperature shifts of the resonances of the NV-center in diamond, Phys. Rev. B 90, 041201 (2014).

[50] C. E. Nebel, M. Stutzmann, F. Lacher, P. Koidl, and R. Zachai, Carrier trapping and release in CVD-diamond films, Diam. Relat. Mater. 7, 556 (1998).

[51] E. Gaubas, T. Ceponis, D. Meskauskaite, and N. Kazuchits, Profiling of current transients in capacitor type diamond sensors, Sensors 15, 13424 (2015). 
[52] N. Mizuochi, T. Makino, H. Kato, D. Takeuchi, M. Ogura, H. Okushi, M. Nothaft, P. Neumann, A. Gali, F. Jelezko, J. Wrachtrup, and S. Yamasaki, Electrically driven singlephoton source at room temperature in diamond, Nat. Photonics, 6, 299 (2012).

[53] H. Kato, M. Wolfer, C. Schreyvogel, M. Kunzer, W. MüllerSebert, H. Obloh, A. Yamasaki, and C. Nebel, Tunable light emission from nitrogen-vacancy centers in single crystal diamond PIN diodes, Appl. Phys. Lett. 102, 151101 (2013).

[54] T. N. Makgato, E. Sideras-Haddad, M. A. Ramos, M. García-Hernández, A. Climent-Font, A. Zucchiatti, A. Muñoz-Martin, S. Shrivastava, and R. Erasmus, Magnetic properties of point defects in proton irradiated diamond, J. Magn. Magn. Mater. 413, 76 (2016).

[55] Y. Sato, T. Shimaoka, J. H. Kaneko, H. Murakami, M. Isobe, M. Osakabe, M. Tsubota, K. Ochiai, A. Chayahara, H. Umezawa, and S. Shikata, Radiation hardness of a single crystal CVD diamond detector for $\mathrm{MeV}$ energy protons, Nucl. Instr. Methods Phys. Res. A 784, 147 (2015).

[56] N. Zhao, S.-W. Ho, and R.-B. Liu, Decoherence and dynamical decoupling control of nitrogen-vacancy center electron spins in nuclear spin baths, Phys. Rev. B 85, 115303 (2012).

[57] F. Kassel, M. Guthoff, A. Dabrowski, and W. de Boer, Description of radiation damage in diamond sensors using an effective defect model, Phys. Status Solidi A 214, 1700162 (2017).

[58] F. Bachmair, et al., (RD42 Collaboration), Diamond sensors for future high energy experiments, Nucl. Instrum. Methods Phys. Res. A 831, 370 (2016).

[59] S. Lagomarsino, S. Sciortino, B. Obreshkov, T. Apostolova, C. Corsi, M. Bellini, E. Berdermann, and C. J. Schmidt, Photoionization of monocrystalline CVD diamond irradiated with ultrashort intense laser pulse, Phys. Rev. B 93, 085128 (2016).

[60] M. Pomorski, B. Caylar, and P. Bergonzo, Super-thin single crystal diamond membrane radiation detectors, Appl. Phys. Lett. 103, 112106 (2013).

[61] K. Bray, H. Kato, R. Previdi, R. Sandstrom, K. Ganesan, M. Ogura, T. Makino, S. Yamasaki, A. P. Magyar, M. Toth, and I. Aharonovich, Single crystal diamond membranes for nanoelectronics, Nanoscale 10, 4028 (2018).

[62] J. Lehnert, J. Meijer, C. Ronning, D. Spemann, and E. Vittone, Ion Beam Induced Charge analysis of diamond diodes, Nucl. Instrum. Methods Phys. Res. B 404, 259 (2017).

[63] H. Umezawa, T. Saito, N. Tokuda, M. Ogura, S. G. Ri, H. Yoshikawa, and S. Shikata, Leakage current analysis of diamond Schottky barrier diode, Appl. Phys. Lett. 90, 073506 (2007).

[64] K. Driche, H. Umezawa, N. Rouger, G. Chicot, and E. Gheeraert, Characterization of breakdown behavior of diamond Schottky barrier diodes using impact ionization coefficients. Jpn. J. Appl. Phys. 56, 04CR12 (2017).

[65] C. Di Venanzio, M. Marinelli, E. Milani, G. Prestopino, C. Verona, G. Verona-Rinati, M. D. Falco, P. Bagalà, R. Santoni, and M. Pimpinella, Characterization of a synthetic single crystal diamond Schottky diode for radiotherapy electron beam dosimetry. Med. Phys. 40, 021712 (2013).

[66] T. Wolf, P. Neumann, K. Nakamura, H. Sumiya, T. Ohshima, J. Isoya, and J. Wrachtrup, Subpicotesla Diamond Magnetometry, Phys. Rev. X 5, 041001 (2015).

[67] T. Unden, P. Balasubramanian, D. Louzon, Y. Vinkler, M. B. Plenio, M. Markham, D. Twitchen, A. Stacey, I. Lovchinsky, A. O. Sushkov, M. D. Lukin, A. Retzker, B. Naydenov, L. P. McGuinness, and F. Jelezko, Quantum Metrology Enhanced by Repetitive Quantum Error Correction, Phys. Rev. Lett. 116, 230502 (2016).

[68] S. Schmitt, T. Gefen, F. M. Stürner, T. Unden, G. Wolff, C. Müller, J. Scheuer, B. Naydenov, M. Markham, S. Pezzagna, J. Meijer, I. Schwarz, M. Plenio, A. Retzker, L. P. McGuinness, and F. Jelezko, Submillihertz magnetic spectroscopy performed with a nanoscale quantum sensor, Science 356, 832 (2017).

[69] B. Naydenov, F. Dolde, L. T. Hall, C. Shin, H. Fedder, L. C. L. Hollenberg, F. Jelezko, and J. Wrachtrup, Dynamical decoupling of a single-electron spin at room temperature, Phys. Rev. B 83, 081201 (2011).

[70] L. P. McGuinness, L. T. Hall, A. Stacey, D. A. Simpson, C. D. Hill, J. H. Cole, K. Ganesan, B. C. Gibson, S. Prawer, and P. Mulvaney, Ambient nanoscale sensing with single spins using quantum decoherence, New J. Phys. 15, 073042 (2013).

[71] F. Picollo, A. Battiato, E. Bernardi, L. Boarino, E. Enrico, J. Forneris, D. Gatto Monticone, and P. Olivero, Realization of a diamond based high density multi electrode array by means of deep ion beam lithography, Nucl. Instr. Methods Phys. Res. B 348, 199 (2015).

[72] F. Dolde, M. W. Doherty, J. Michl, I. Jakobi, B. Naydenov, S. Pezzagna, J. Meijer, P. Neumann, F. Jelezko, N. B. Manson, and J. Wrachtrup, Nanoscale Detection of a Single Fundamental Charge in Ambient Conditions Using the NVCenter in Diamond, Phys. Rev. Lett. 112, 097603 (2014).

[73] S. Castelletto, B. C. Johnson, V. Ivády, N. Stavrias, T. Umeda, A. Gali, and T. Ohshima, A silicon carbide roomtemperature single-photon source, Nat. Mater. 13, 151 (2014).

[74] F. Bosia, N. Argiolas, M Bazzan, B. A. Fairchild, A. D. Greentree, D. W. M. Lau, P. Olivero, F. Picollo, S. Rubanov, and S. Prawer, Direct measurement and modelling of internal strains in ion-implanted diamond, J. Phys.: Condens. Matter 25, 385403 (2013).

[75] A. Battiato, M. Lorusso, E. Bernardi, F. Picollo, F. Bosia, D. Ugues, A. Zelferino, A. Damin, J. Baima, N. M. Pugno, E. P. Ambrosio, and P. Olivero, Softening the ultra-stiff: Controlled variation of Young's modulus in single-crystal diamond, Acta Mater. 116, 95 (2016).

[76] Y. X. Wei, R. J. Wang, and W. H. Wang, Soft phonons and phase transition in amorphous carbon, Phys. Rev. B 72, 012203 (2005).

[77] B. A. Fairchild, S. Rubanov, D. W. M. Lau, M. Robinson, I. Suarez-Martinez, N. Marks, A. D. Greentree, D. McCulloch, and S. Prawer. Mechanism for the amorphisation of diamond, Adv. Mater. 24, 2024 (2012). 